J. Lake Sci. (湖泊科学) , 2012, 24(6): 914-922

http: //www.jlakes.org. E-mail : jlakes@niglas.ac.cn

(c) 2012 by Journal of Lake Sciences

\title{
太湖有色溶解有机物对水体总吸收贡献的遥感估算”
}

\author{
姜广甲 $^{1,2}$, 马荣华 ${ }^{1 * *}, \mathbf{E}^{\text {洪涛 }}{ }^{1}$ \\ (1: 中国科学院南京地理与湖泊研究所湖泊与环境国家重点实验室, 南京 210008) \\ (2:中国科学院研究生院, 北京 100049)
}

\begin{abstract}
摘 要: 有色溶解有机物 ( CDOM) 是决定自然水体水色的主要溶解物质, 其吸光能力和光化降解产物对水体初级生产力 和碳循环过程具有重要影响. 以太湖为研究区,2004 年 10 月、2008 年 10 月、2010 年 4 月和 2011 年 1 月和 3 月共 5 期实测 数据, 采集了 333 个有效样点, 分析不同时期 CDOM 对水体总吸收的贡献, 并利用遥感技术估算 $\left[a_{\mathrm{CDOM}} / a_{\mathrm{t}}\right](412)$. 结果 表明:不同时期 $\left[a_{\mathrm{CDOM}} / a_{\mathrm{t}}\right]$ (412) 均值变化明显, 2011 年 $\left[a_{\mathrm{CDOM}} / a_{\mathrm{t}}\right]$ ( 412$)$ 的均值最大 (0.369), 高于所有样点 $\left[a_{\mathrm{CDOM}} / a_{\mathrm{t}}\right](412)$ 均值 $(0.295 \pm 0.139) ; 201004$ 期的 $\left[a_{\mathrm{CDOM}} / a_{\mathrm{t}}\right]$ (412) 在 $0.046 \sim 0.455$ 之间变化,其均值最小 $(0.236 \pm$ $0.108) ; 200410$ 和 200810 两期数据 $\left[a_{\mathrm{CDOM}} / a_{\mathrm{t}}\right](412)$ 均值相差不大. 竺山湾、梅梁湾与整个太湖相比,竺山湾 $\left[a_{\mathrm{CDOM}} / a_{\mathrm{t}}\right]$ (412) 均值较高, 对太湖 $\left[a_{\mathrm{CDOM}} / a_{\mathrm{t}}\right]$ (412) 的贡献较大, 而梅梁湾 $\left[a_{\mathrm{CDOM}} / a_{\mathrm{t}}\right]$ (412) 均值与整个太湖相差较小. 利用多元线 性模型估算 $\left[a_{\mathrm{CDOM}} / a_{\mathrm{t}}\right]$ (412) 精度较高 $(n=333, R M S E=34.60 \%)$. 悬浮泥沙和浮游色素是影响 $\left[a_{\mathrm{CDOM}} / a_{\mathrm{t}}\right](412)$ 遥感估 算精度的主要原因, 浮游色素的吸收造成 $\left[a_{\mathrm{CDOM}} / a_{\mathrm{t}}\right](412)$ 的值被低估, 而悬浮泥沙的吸收使得 $\left[a_{\mathrm{CDOM}} / a_{\mathrm{t}}\right](412)$ 的值被 高估,并且悬浮泥沙是影响 CDOM 吸收的主要原因.
\end{abstract}

关键词: CDOM; 遥感; 碳循环; 太湖; 内陆水体

\section{Estimation of the contribution of chromophoric dissolved organic matter to total light ab- sorption by remote sensing in Lake Taihu}

\author{
JIANG Guangjia ${ }^{1,2}$, MA Ronghua ${ }^{1} \&$ DUAN Hongtao ${ }^{1}$ \\ (1: State Key Laboratory of Lake Science and Environment, Nanjing Institute of Geography and Limnology, Chinese Academy \\ of Sciences, Nanjing 210008, P. R. China) \\ (2: Graduate University of Chinese Academy of Sciences, Beijing 100049, P. R. China)
}

Abstract: Chromophoric dissolved organic matter ( CDOM) mainly absorbs light in water which may influence the nature water color in lakes. Its absorption and photochemical degradation products play an important role in the primary productivity of water and carbon cycle. In Lake Taihu, a total of 333 sites were sampled in October 2004, October 2008, April 2010 and January and March 2011 to analyze the contribution of CDOM to total light absorption and estimate $\left[a_{\mathrm{CDOM}} / a_{\mathrm{t}}\right](412)$ from remote sensing. It was found that the average of $\left[a_{\mathrm{CDOM}} / a_{\mathrm{t}}\right](412)$ exhibited highly temporal variations during the five cruises. The maximum (0.369) was determined in 2011, comparing with all samples in Lake Taihu $(0.295 \pm 0.139)$. The minimum average of [ $\left.a_{\mathrm{CDOM}} / a_{\mathrm{t}}\right]$ (412) in the dataset 201004 was $0.236 \pm 0.108$, varing from 0.046 to 0.455 . No significant difference was observed in the dataset 200410 and 200810. The mean of $\left[a_{\mathrm{CDOM}} / a_{\mathrm{t}}\right](412)$ in Zhushan Bay was higher than that in both whole Lake Taihu and Meiliang Bay. For Meiliang Bay, it had almost the same value with the whole lake. A multi-band algorithm was adopted to estimate the $\left[a_{\mathrm{CDOM}} / a_{\mathrm{t}}\right](412)$ by remote sensing and acceptable results were detected $(n=333, R M S E=34.60 \%)$. Suspended sediments and pigments had an important impact on determination of $\left[a_{\mathrm{CDOM}} / a_{\mathrm{t}}\right](412)$ from remote sensing. It was underestimated because of pigments and overestimated as the suspended sediments in water and the latter was worse. The results also showed that the CDOM and detritus optically dominate the water color in Lake Taihu.

Keywords: CDOM; remote sensing; carbon cycle; Lake Taihu; inland water

\footnotetext{
* 中国科学院知识创新工程重要方向性项目 (KZCX2-YW-QN311, KZCX2-EW-QN308) 和国家自然科学基金项目 (41171271,41171273) 联合资助. 2011-12-08 收稿;2012-02-21 收修改稿. 姜广甲,男,1984 年生, 博士研究生; E-mail : gjjiang2011@ gmail. com.

** 通信作者;E-mail:mrhua2002@ niglas. ac. cn.
} 
有色溶解有机物 (Chromophoric Dissolved Organic Matter, CDOM) 是水体中溶解有机物 (DOM) 的重要组 成部分, 是决定自然水体水色的主要溶解物质 ${ }^{[1-2]}$. CDOM 的光学特性主要表现为吸收, 在紫外波段更为强 烈 (UVC、UVB 和 UVA), 从而影响着水体近表面光传输过程. 同时, CDOM 在可见光波段也有较强的吸收, 影响水体离水辐亮度, 为 $\mathrm{CDOM}$ 的遥感反演奠定了物理基础 ${ }^{[3]}$. 内陆水体 (如湖泊、水库以及河口等) 中 CDOM 主要有 2 个来源, 一是受陆源影响, 主要是陆生植被腐烂降解后由河流携带进人水体 ${ }^{[4]}$; 二是一些生 物过程如细菌降解、浮游植物等腐烂的产物 ${ }^{[5]}$. CDOM 的组成成分复杂, 是由氨基酸、糖类、脂肪酸类和酚类 等物质组成的复杂混合物 ${ }^{[6]}$.

$\mathrm{CDOM}$ 在水体碳循环中担任重要角色 ${ }^{[7]}$. 经呼吸作用和光化降解后, $\mathrm{CDOM}$ 可以分解为 $\mathrm{CO}_{2}$ 和 $\mathrm{CO}^{[8]}$. CDOM 在 UVB、UVA 和 PAR 波段内的光化降解能力取决于光量子产率和水体中的人射光能 ${ }^{[9]}$. 其光化降解 能力直接决定了水体中无机碳的释放量, 进而影响大气中温室气体的含量. 因此, 充分了解 CDOM 对水体总 吸收的贡献率具有十分重要的生态意义.

内陆水体中, 影响水体光学特性主要有 4 种物质: 水体本身、浮游植物、悬浮泥沙和 $\mathrm{CDOM}^{[10-11]}$. 水体中 高浓度的悬浮泥沙 (Nonalgal Particles, NAP) 直接影响光在水体中的传播, 决定光的能量和再分配过程, 进 而影响水体透明度、真光层深度、水色等光学性质, 对 CDOM 的光化降解和水下浮游植物的光合作用有重要 影响. 悬浮泥沙与 CDOM 具有相似的吸光性质 ${ }^{[4]}$, 即随波长增加, 吸收系数呈指数递减. 利用遥感技术直接 估算水体中 CDOM 含量比较困难, 大量学者往往将两者合并为非色素物质 (即, CDM $=$ CDOM $+\mathrm{NAP}$ ) 进行 遥感估算分析. 利用 CDOM 对水体总吸收的贡献有助于将 CDOM 从 CDM 中分离出来.

遥感具有快速、大范围、周期性的特点, 在湖泊水色监测中发挥重要的作用 ${ }^{[12]}$. 中分辨率成像光谱仪 (Moderate Resolution Imaging Spectroradiometer, MODIS) 具有 36 个光谱通道, 分布在 $0.4 \sim 14.3 \mu \mathrm{m}$ 范围内, 多波段数据可以同时提供反映陆地、云边界, 云特性, 海洋水色、浮游植物、生物地理、化学, 大气中水汽, 地 表、云顶温度, 大气温度, 臭氧和云顶高度等特征的信息; 有 $250 、 500$ 和 $1000 \mathrm{~m}$ 三种空间分辨率; 时间分辨率 高, 每 $1 \sim 2 \mathrm{~d}$ 可获得一次全球观测数据. 因此, MODIS 数据已广泛应用于湖泊水质遥感监测中 ${ }^{[13]}$.

针对太湖 ${ }^{[14-16]}$ 、青海湖 ${ }^{[17]}$ 、巢湖 ${ }^{[18]}$ 、龙感湖 ${ }^{[19]}$ 和石头口门水库 ${ }^{[20]}$ 等内陆水体, 国内学者对 CDOM 光学 特性以及季节性变化规律等方面做了大量研究. 本文以太湖为研究区域, 旨在分析太湖 CDOM 对水体总吸 收贡献的变化; 利用实测光谱数据和 MODIS 影像资料估算 CDOM 吸收对水体总吸收的贡献; 评价 CDOM 对 水体总吸收贡献的遥感估算精度.

\section{1 材料与方法}

\section{1 样点布设}

2004 年 10 月 (200410)、2008 年 10 月(200810)、2010 年 4 月 (201004)、2011 年 1 月 (201101) 和 2011 年 3 月 (201103) 在太湖共布设 333 个采样点进行水体反射光谱测量 (图 1), 并同步进行 GPS 定位, 测定各样点透明度、水深等常规数据. 采样点信息见表 1 . 采集表层水样后放人苯板保温箱保存, 运回实验室在 $4^{\circ} \mathrm{C}$ 下冷藏, 温度达室温后测定水质参数.

所用光谱仪为美国 ASD 公司生产的 FieldSpec Pro Dual VNIR 野外双通道光谱仪, 可在 $350 \sim 1050 \mathrm{~nm}$ 波段范 围内连续测量, 测量方法采用水面之上测量法 ${ }^{[21]}$, 测定水体、灰板以及天空光光谱, 通过计算得到水体反射率.

\section{2 参数测量}

利用 $10 \%$ 的盐酸浸泡后的 Whatman 聚碳酸酯滤膜 (孔径 $0.22 \mu \mathrm{m}$ ) 过滤水样, 得到各样点滤液. 以 Milli-Q水为参比, 利用 UV-2401 分光光度计 (日本岛津) 在 $200 \sim 800 \mathrm{~nm}$ (1 nm 间隔) 波段范围内扫描测定滤 液的吸光度, 通过计算得到 CDOM 的吸收系数(公式(1)), 并对 CDOM 吸收系数进行散射校正 (公式 (2)), 从而得到校正后的 CDOM 的吸收光谱 ${ }^{[4]}$.

$$
\begin{gathered}
a_{\mathrm{CDOM}}(\lambda)^{\prime}=2.303 D(\lambda) / l \\
a_{\mathrm{CDOM}}(\lambda)=a_{\mathrm{CDOM}}(\lambda)^{\prime}-a_{\mathrm{CDOM}}(\lambda)^{\prime} \cdot \lambda / 700
\end{gathered}
$$

式中, $\lambda$ 为波长 $(\mathrm{nm}), a_{\mathrm{CDOM}}(\lambda)^{\prime}$ 为未校正的吸收系数 $\left(\mathrm{m}^{-1}\right), D(\lambda)$ 为吸光度, $l$ 为光程路径 $(\mathrm{m}), a_{\mathrm{CDOM}}(\lambda)$ 为 校正后吸收系数. 


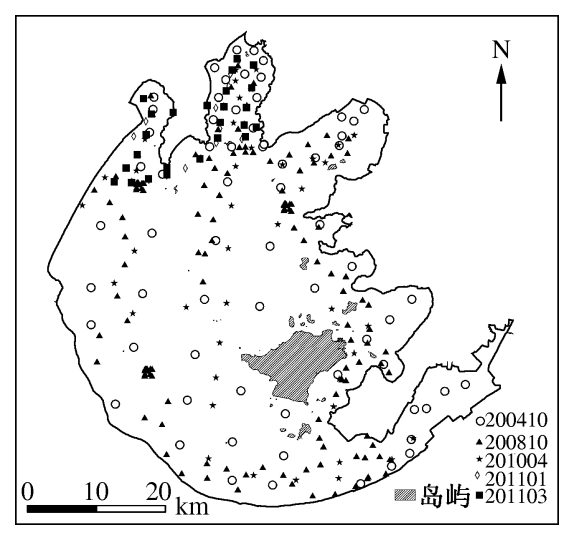

图 1 太湖采样点分布 (在 5 次采样中,梅梁湾、竺山湾区域为常规采样部分,不同期采样的样点重合)

Fig. 1 Distribution of sampling sites in Lake Taihu

表 1 太湖采样点信息

Tab. 1 Information of sampling sites in Lake Taihu

\begin{tabular}{ccccc}
\hline 采样时间 & 编号 & 样点个数 & 采样区域 & $a_{\mathrm{CDOM}}(412)$ 均值 $/ \mathrm{m}^{-1}$ \\
\hline $2004-10-18-10-29$ & 200410 & 65 & 全太湖 & $1.45 \pm 0.50$ \\
$2008-10-06-10-17$ & 200810 & 145 & 全太湖 & $1.25 \pm 0.39$ \\
$2010-04-23-05-12$ & 201004 & 78 & 全太湖 & $0.99 \pm 0.41$ \\
$2011-01-22-01-23$ & 201101 & 19 & 竺山湾、梅梁湾 & $1.79 \pm 0.77$ \\
$2011-03-24-03-25$ & 201103 & 26 & 䇥山湾、梅梁湾 & $1.69 \pm 0.91$ \\
\hline
\end{tabular}

使用 Whatman 生产的 GF/C 膜过滤水样. 利用 UV-2401 分光光度计 (日本岛津) 测定总颗粒物吸光度, 波长范围为 $350 \sim 800 \mathrm{~nm}$, 分差 $1 \mathrm{~nm}$, 然后计算得到总颗粒物吸收系数 ${ }^{[22]}$. 将附有总颗粒物的滤膜利用丙酮 浸泡 30 60 min 去除总颗粒物中的色素成分, 根据同样 (总颗粒物吸收) 的方法测定非色素颗粒物吸收系 数. 将总颗粒物吸收系数减去非色素颗粒物吸收系数即可得到色素颗粒物吸收系数.

\section{3 模型构建}

水体光学特性包括固有光学特性 (Inherent Optic Properties, IOPs) 和表观光学特性(Apparent Optic Properties, AOPs $)^{[13]}$. 水体的生物光学模型将两者有机地联系在一起.

恰好位于水体表面之下的漫反射比 $R\left(\lambda, 0^{-}\right)$可表示为:

$$
R\left(\lambda, 0^{-}\right)=\frac{E_{\mathrm{u}}\left(0^{-}\right)}{E_{\mathrm{d}}\left(0^{-}\right)}=f \frac{b_{\mathrm{b}}(\lambda)}{a(\lambda)+b_{\mathrm{b}}(\lambda)}
$$

式中, $0^{-}$表示恰好位于水面以下; $E_{\mathrm{u}}\left(0^{-}\right)$指向上辐照度, $E_{\mathrm{d}}\left(0^{-}\right)$指向下辐照度; $f$ 与太阳高度角有关, $a(\lambda)$ 和 $b_{\mathrm{b}}(\lambda)$ 分别为吸收系数和后向散射系数.

而 $E_{\mathrm{u}}\left(0^{-}\right)=Q n^{2} / t \cdot L_{\mathrm{w}}(\lambda) ; E_{\mathrm{d}}\left(0^{-}\right)=(1-\rho) E_{\mathrm{d}}\left(0^{+}\right) ; R_{\mathrm{rs}}(\lambda)=L_{\mathrm{w}}(\lambda) / E_{\mathrm{d}}\left(0^{+}\right) . Q$ 为光场分布函数; $\rho$ 为气水表面辐照度反射率; $n$ 为水体折射率; $t$ 为气一水表面透射率; $R_{\mathrm{rs}}(\lambda)$ 为遥感反射比. 因此:

$$
R_{\mathrm{rs}}(\lambda)=\frac{f t(1-\rho)}{Q n^{2}} \cdot \frac{b_{\mathrm{b}}(\lambda)}{a_{\mathrm{t}}(\lambda)+b_{\mathrm{b}}(\lambda)}
$$

水体总吸收系数 $\left(a_{\mathrm{t}}(\lambda)\right)$ 为各物质组分吸收系数的贡献和, 公式为:

$$
a_{\mathrm{t}}(\lambda)=a_{\mathrm{w}}(\lambda)+a_{\mathrm{CDOM}}(\lambda)+a_{\mathrm{ph}}(\lambda)+a_{\mathrm{d}}(\lambda)
$$

式中, $a_{\mathrm{w}}(\lambda)$ 为纯水的吸收系数 ${ }^{[23-24]} ; a_{\mathrm{CDOM}}(\lambda)$ 为 CDOM 的吸收系数; $a_{\mathrm{ph}}(\lambda)$ 为浮游植物色素的吸收系数; $a_{\mathrm{d}}(\lambda)$ 为悬浮泥沙的吸收系数. 
水体总后项散射系数可以表示为水体各组分后项散射系数的线性和,即:

$$
b_{\mathrm{b}}(\lambda)=b_{\mathrm{bw}}(\lambda)+b_{\mathrm{bp}}(\lambda)
$$

式中, $b_{\mathrm{bw}}(\lambda)$ 为纯水的后向散射系数; $b_{\mathrm{bp}}(\lambda)$ 表示总悬浮物后向散射系数.

CDOM 在紫外和可见光波段范围内以吸收为主, 在 $412 \mathrm{~nm}$ 处吸收较强烈, 其散射效应可忽略不计 ${ }^{[25]}$; 悬浮泥沙吸收特性与 CDOM 相似,但悬浮颗粒物在 $555 \mathrm{~nm}$ 处具有较强的后向散射作用; 浮游植物色素在蓝 波段内吸收比较强烈, 并且在 $490 \mathrm{~nm}$ 处吸收最强烈 ${ }^{[3]}$. 考虑到浮游植物和悬浮泥沙对 CDOM 吸收的影响, 选取 $R_{\mathrm{rs}}(412) 、 R_{\mathrm{rs}}(490)$ 和 $R_{\mathrm{rs}}(555)$ 建立 CDOM 对水体总吸收贡献的遥感估算模型 (CDOM 对水体总吸收 的贡献表示为: $\left.\left[a_{\mathrm{CDOM}} / a_{\mathrm{t}}\right](412)\right)$.

\section{4 模型评价方法}

利用遥感技术估算太湖水体 CDOM 吸收对水体总吸收的贡献, 利用现场数据对模型的可靠性与鲁棒性 进行验证和分析. 采用观测值与模拟值之间的均方根误差 (Root Mean Square Error, RMSE) 来评价估算模型 的优劣,其具体表现形式如下 ${ }^{[26]}$ :

$$
R M S E=\sqrt{\frac{\sum_{i=1}^{n}\left(P_{i}-O_{i}\right)^{2}}{n}} \times \frac{100}{\bar{O}}
$$

式中, $P_{i}$ 和 $O_{i}$ 分别为模拟值和观测值; $\bar{O}$ 为观测值的平均值; $n$ 为样本数.

\section{5 数据处理与分析}

数据统计、多元线性拟合等数据处理均在 Origin 8.0 统计软件中完成.

\section{2 结果与分析}

\section{1 太湖 $\left[a_{\mathrm{CDOM}} / a_{\mathrm{t}}\right](412)$ 的时空分布规律}

太湖不同采样期 CDOM 对水体总吸收的贡献 见图 2.2011 年两期数据 $\left[a_{\mathrm{CDOM}} / a_{\mathrm{t}}\right](412)$ 的均值最 大 $(0.37)$, 高于所有样点均值 $(0.30 \pm 0.14)$; 201004 的 $\left[a_{\mathrm{CDOM}} / a_{\mathrm{t}}\right](412)$ 在 $0.05 \sim 0.46$ 之间变 化,均值最小 $(0.24 \pm 0.11) ; 200410$ 和 200810 两期 数据均值相差不大 (分别为 $0.30 \pm 0.12$ 和 $0.31 \pm 0.16$ ), 但 200810 各样点间差异显著 (标准 差为 0.16$)$, 变化范围为 $0.02 \sim 0.71$. 所有样点的 $\left[a_{\mathrm{CDOM}} / a_{\mathrm{t}}\right](412)$ 均值都低于河口、海岸带水域的 研究结果 $(0.55 \pm 0.15)^{[3]}$.

2011 年两次采样主要集中在竺山湾和梅梁湾 两个区域 (表 1 ), 为了进行统一比较, 针对竺山湾 和梅梁湾两个区域进行比较 (图 3).

竺山湾 $\left[a_{\mathrm{CDOM}} / a_{\mathrm{t}}\right](412)$ 均值高于梅梁湾 (200810 期数据除外), 其中, 201103 期数据相差最 大, 差值为 $0.222 ; 201004$ 期相差最小 (差值为 $0.020)$. 针对梅梁湾而言, 201101 期数据 $\left[a_{\mathrm{CDOM}} / a_{\mathrm{t}}\right]$ (412) 均值最大 $(0.38 \pm 0.12), 201103$ 期最小 $(0.28 \pm 0.07)$, 其他年份均值相差不大. 对于竺山湾 来说, 201103 期数据 $\left[a_{\mathrm{CDOM}} / a_{\mathrm{t}}\right](412)$ 均值最大 $(0.50 \pm 0.11)$, 是 200810 期 $\left[a_{\mathrm{CDOM}} / a_{\mathrm{t}}\right](412)$ 均值 的 1.7 倍 $(0.29 \pm 0.03)$, 其次是 201101 期数据

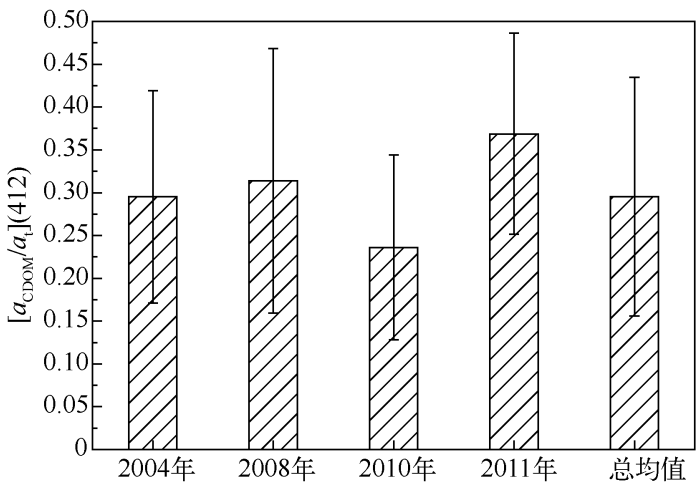

图 2 不同年份 CDOM 对水体总吸收的贡献 (均值)

Fig. 2 Contribution of CDOM to total light absorption in different years

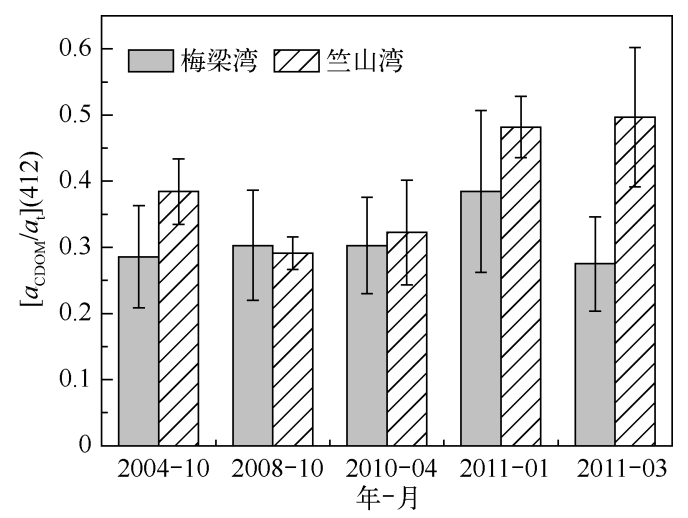

图 3 梅梁湾和答山湾不同采样 日期 CDOM 对水体总吸收的贡献

Fig. 3 Contribution of CDOM to total light absorption in different sampling dates in the area of Meiliang Bay and Zhushan Bay 
$(0.48 \pm 0.05)$, 再者是 200410 期 $(0.38 \pm 0.05), 201004$ 和 200810 两期数据 $\left[a_{\mathrm{CDOM}} / a_{\mathrm{t}}\right]$ (412) 均值相差不大.

竺山湾、梅梁湾与整个太湖相比, 笈山湾 $\left[a_{\mathrm{CDOM}} / a_{\mathrm{t}}\right]$ (412) 均值较高, 对太湖 $\left[a_{\mathrm{CDOM}} / a_{\mathrm{t}}\right](412)$ 的贡献较 大,而梅梁湾 $\left[a_{\mathrm{CDOM}} / a_{\mathrm{t}}\right](412)$ 均值与整个太湖相差不大 (图 2、图 3).

\section{2 估算模型}

水体光谱反射率受水体组分浓度的影响, 水色遥感的最终目的是建立遥感反射率或离水辐亮度与水体 组分浓度之间的关系反演水色参数浓度 ${ }^{[13]}$. 内陆水体光谱反射率由纯水、浮游植物、悬浮泥沙和 CDOM 共 同决定,不同物质具有不同的波谱响应,并且各组分之间相互影响.

为估算 CDOM 吸收占水体总吸收的比率, 本研究选取 $R_{\mathrm{rs}}(412) 、 R_{\mathrm{rs}}(490)$ 和 $R_{\mathrm{rs}}(555)$ 建立多元线性模 型估算 $\left[a_{\mathrm{CDOM}} / a_{\mathrm{t}}\right](412)$, 其模型形式为:

$$
\left[a_{\mathrm{CDOM}} / a_{\mathrm{t}}\right](412)=\alpha+\beta \cdot R_{\mathrm{rs}}(412)+\gamma \cdot R_{\mathrm{rs}}(490)+\delta \cdot R_{\mathrm{rs}}(555)
$$

式中, $\alpha, \beta 、 \gamma$ 和 $\delta$ 为回归系数.

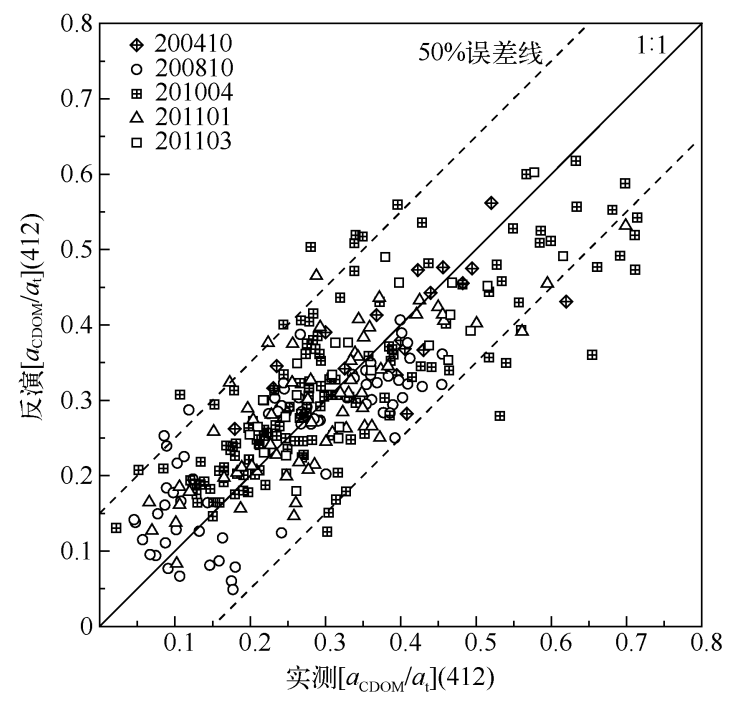

图 $4\left[a_{\mathrm{CDOM}} / a_{\mathrm{t}}\right](412)$ 实测值与反演值的关系

Fig. 4 Comparison between measured and estimated

$$
\left[a_{\mathrm{CDOM}} / a_{\mathrm{t}}\right](412)
$$

太湖不同时期 $a_{\mathrm{CDOM}} / a_{\mathrm{t}}$ 遥感估算的回归系数、 决定性系数 $\left(R^{2}\right)$ 和 $R M S E$ (表 2 ) 表明, 不同时期 数据的反演精度差异较大, 201103 期数据反演精 度最高 $\left(R^{2}=0.68, R M S E=19.18 \%\right), 200410$ 、 200810 和 201004 期数据反演效果较好, 决定系数 均在 0.60 以上, 201101 期数据效果最差, 决定系 数为 0.50 , 但 $R M S E$ 最小 $(19.20 \%)$. 所有样点参 与遥感估算过程时, 反演精度有所降低 $\left(R^{2}=\right.$ $0.48, R M S E=34.60 \%)$; 不同时期多元线性拟合 得到的 4 个回归系数, 同一系数值差异较大, 但具 有相同趋势: $\alpha$ 均为正值, $\beta$ 为负值, $\gamma$ 为正值, $\delta$ 为 负值.

实测 $\left[a_{\mathrm{CDOM}} / a_{\mathrm{t}}\right](412)$ 和反演 $\left[a_{\mathrm{CDOM}} / a_{\mathrm{t}}\right]$ (412) 的关系显示 (图 4), 各点均匀分布于 $1: 1$ 线 左右, 说明利用公式 (8) 估算太湖 CDOM 对水体 总吸收的贡献效果较好.

\section{3 模型验证与评价}

利用多元线性模型估算太湖水体 $\left[a_{\mathrm{CDOM}} / a_{\mathrm{t}}\right]$ (412) 的精度较高, 该模型适合太湖水体 CDOM 对水体总吸收贡献的遥感反演. 201101 和 201103

期数据采样区域是梅梁湾和竺山湾, 而其他 3 期数据在全太湖采样 (表 1 ). 为了验证模型在空间上的稳定 性, 建立 200410 期、200810 期和 201004 期梅梁湾和竺山湾水域样点的 $\left[a_{\mathrm{CDOM}} / a_{\mathrm{t}}\right]$ (412) 估算模型, 并将模型 系数应用于全湖样点数据, 以评价模型在太湖不同区域鲁棒性 (表 3 ).

表 2 太湖不同时期 $a_{\mathrm{CDOM}} / a_{\mathrm{t}}(412)$ 遥感估算的回归系数、决定性系数和 $R M S E$

Tab. 2 Regression coefficients, determination coefficients and RMSE of estimation of $a_{\mathrm{CDOM}} / a_{\mathrm{t}}(412)$ by remote sensing in different sampling dates in Lake Taihu

\begin{tabular}{ccccrrrc}
\hline 航次 & 样点个数 & $\alpha$ & $\beta$ & $\gamma$ & \multicolumn{1}{c}{$\delta$} & $R^{2}$ & $R M S E / \%$ \\
\hline 200410 & 65 & 0.52 & -25.61 & 13.57 & -5.05 & 0.63 & 25.48 \\
200810 & 145 & 0.66 & -19.33 & 4.91 & -7.69 & 0.62 & 30.02 \\
201004 & 78 & 0.51 & -53.91 & 39.29 & -15.27 & 0.61 & 30.03 \\
201101 & 19 & 0.69 & -42.99 & 45.12 & -21.76 & 0.50 & 19.20 \\
201103 & 26 & 0.84 & -37.22 & 61.55 & -48.29 & 0.68 & 19.18 \\
所有样点 & 333 & 0.53 & -35.13 & 12.11 & -1.70 & 0.48 & 34.60 \\
\hline
\end{tabular}


表 3 梅梁湾和竺山湾 $\left[a_{\mathrm{CDOM}} / a_{\mathrm{t}}\right]$ (412) 实测值与估算值之间的回归系数

Tab. 3 Regression coefficients between measured and estimated $\left[a_{\mathrm{CDOM}} / a_{\mathrm{t}}\right](412)$ in Meiliang Bay and Zhushan Bay

\begin{tabular}{cccccc}
\hline 航次 & 样点个数 & 梅梁湾、筷山湾样点个数 & 截距 & 斜率 & 差值平均值 $\Delta^{*}$ \\
\hline 200410 & 65 & 24 & 0.136 & 0.469 & 0.017 \\
200810 & 145 & 9 & 0.260 & 0.296 & -0.033 \\
201004 & 78 & 52 & 0.227 & 0.255 & -0.042 \\
\hline
\end{tabular}

*差值平均值: $\Delta=\frac{1}{N} \cdot \sum_{1}^{N}\left(\left[a_{\mathrm{CDOM}} / a_{\mathrm{t}}\right]^{\mathrm{Est}}-\left[a_{\mathrm{CDOM}} / a_{\mathrm{t}}\right]^{\mathrm{Mea}}\right)$, 式中 $\left[a_{\mathrm{CDOM}} / a_{\mathrm{t}}\right]^{\mathrm{Est}}$ 为估算值, $\left[a_{\mathrm{CDOM}} / a_{\mathrm{t}}\right]^{\mathrm{Mea}}$ 为实测值.

$\Delta$ 值均控制在 \pm 0.042 范围之内, 说明模型形式和拟合系数具有一定的稳定性, 适合太湖水体. 因此, 利 用梅梁湾、竺山湾的回归系数可以估算整个太湖的 $\left[a_{\mathrm{CDOM}} / a_{\mathrm{t}}\right](412)$.

\section{4 MODIS 遥感影像 $\left[a_{\mathrm{CDOM}} / a_{\mathrm{t}}\right](412)$ 估算}

基于太湖 333 个实测样点数据,利用 2004 年 10 月 24 日、2008 年 10 月 15 日、2010 年 4 月 30 日、 2011 年 1 月 25 日和 3 月 23 日的 MODIS 遥感影像, 空间分辨率为 $1 \mathrm{~km}$. 经条带去除、大气校正 (FLAASH 模型) 和几何粗校正后, 选取 MODIS 的 8 波段( $405 \sim 420 \mathrm{~nm}$, 中心波长 $412.5 \mathrm{~nm}$ )、10 波段 (483 493 nm, 中心波长 $488 \mathrm{~nm}$ ) 和 12 波段 (546 $556 \mathrm{~nm}$, 中心波长 $551 \mathrm{~nm})$, 利用表 2 中的系数估算 太湖水体 $\left[a_{\mathrm{CDOM}} / a_{\mathrm{t}}\right](412)$.

将 MODIS 遥感影像 $\left[a_{\mathrm{CDOM}} / a_{\mathrm{t}}\right]$ (412) 估算值与实 测值进行对比, 发现两者具有较好的相关性( 图 5 ), 样点均匀地分布在 1:1 线的周围, 表明 $\left[a_{\mathrm{CDOM}} / a_{\mathrm{t}}\right]$ (412) 的估算效果较好, 该模型适于太湖水体. 进而 说明利用 MODIS 影像数据能客观反映 $\left[a_{\mathrm{CDOM}} / a_{\mathrm{t}}\right]$ (412) 的时空变化规律.

太湖东太湖区域水生植被较多, 很大程度上影 响了水体的离水辐亮度, 对遥感影像反射率影响较 大, 故剔除东太湖区域水体. MODIS 遥感影像反映

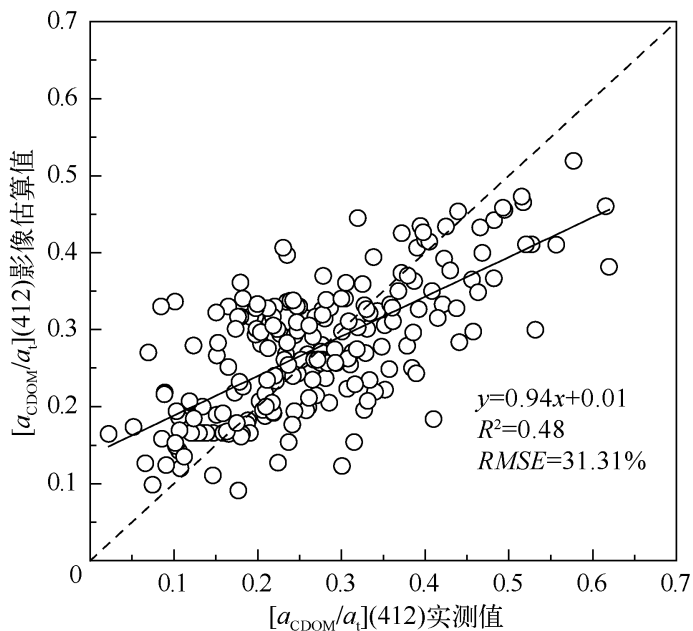

图 5 MODIS 遥感影像 $\left[a_{\mathrm{CDOM}} / a_{\mathrm{t}}\right]$ (412)估算值与 实测值之间的关系

Fig. 5 Relationship between the estimation by MODIS and measured data for $\left[a_{\mathrm{CDOM}} / a_{\mathrm{t}}\right](412)$ 了 $\left[a_{\mathrm{CDOM}} / a_{\mathrm{t}}\right](412)$ 在空间和时间上的动力变化过 程 (图 6). 整体上看, 太湖南部 CDOM 吸收对水体总吸收的贡献较小, 再者是太湖中心区域; 梅梁湾、竺山湾 和贡湖湾 3 个区域 $\left[a_{\mathrm{CDOM}} / a_{\mathrm{t}}\right](412)$ 值较大; 太湖西部的 $\left[a_{\mathrm{CDOM}} / a_{\mathrm{t}}\right](412)$ 值随时间变化显著.

\section{3 讨论}

CDOM 的吸收受悬浮泥沙和浮游藻类的影响, 导致 CDOM 吸收占水体总吸收的比例有不同变化. 本文 着重分析两者对 CDOM 吸收的影响,并讨论两者对 CDOM 吸收贡献影响的主导因素.

浮游植物色素在 $490 \mathrm{~nm}$ 处吸收强烈,而悬浮泥沙在 $555 \mathrm{~nm}$ 的后向散射最强. 利用 $a_{\mathrm{ph}}(490) / R_{\mathrm{rs}}(555)$ 来评估悬浮泥沙和浮游色素对 $\left[a_{\mathrm{CDOM}} / a_{\mathrm{t}}\right](412)$ 遥感估算结果的影响, 即建立 $a_{\mathrm{ph}}(490) / R_{\mathrm{rs}}(555)$ 与 $\Delta\left[a_{\mathrm{CDOM}} / a_{\mathrm{t}}\right]$ (412) 之间的相关关系, 其中, $\Delta\left[a_{\mathrm{CDOM}} / a_{\mathrm{t}}\right]$ (412) 为实测值与估算值之间的差值.

$a_{\mathrm{ph}}(490) / R_{\mathrm{rs}}(555)$ 与 $\Delta\left[a_{\mathrm{CDOM}} / a_{\mathrm{t}}\right]$ (412) 的关系表明（图 7 ), 两者有明显的反比关系, 即 $\Delta\left[a_{\mathrm{CDOM}} / a_{\mathrm{t}}\right]$ (412) 为负值时, $a_{\mathrm{ph}}(490) / R_{\mathrm{rs}}(555)$ 的值较大, 说明浮游色素的吸收对 $\left[a_{\mathrm{CDOM}} / a_{\mathrm{t}}\right](412)$ 遥感估 算影响比较大, 并且 $\left[a_{\mathrm{CDOM}} / a_{\mathrm{t}}\right](412)$ 的值被低估; 反之, 悬浮泥沙的吸收对 $\left[a_{\mathrm{CDOM}} / a_{\mathrm{t}}\right](412)$ 遥感估算影响 


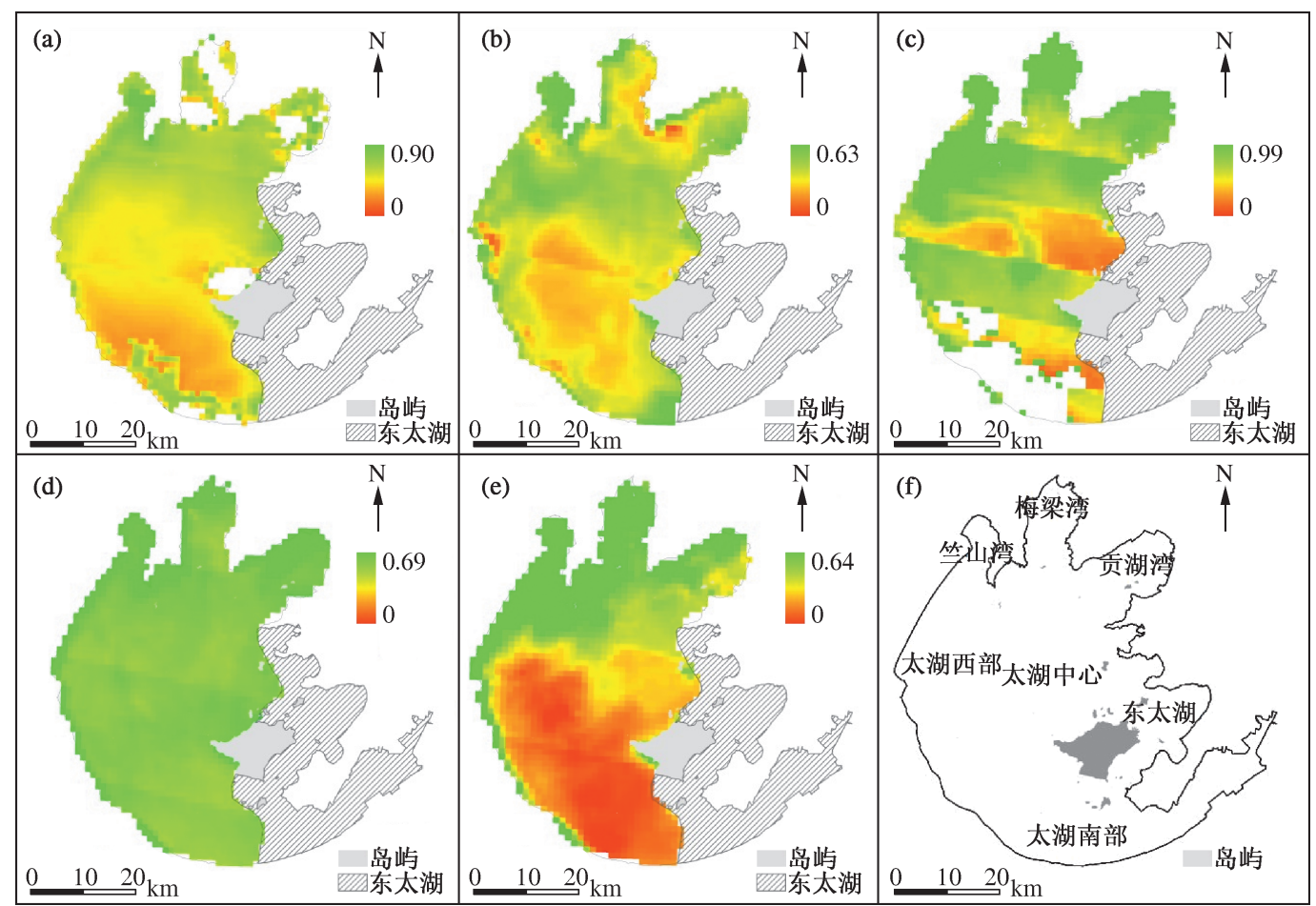

图 6 MODIS 遥感影像 $\left[a_{\mathrm{CDOM}} / a_{\mathrm{t}}\right](412)$ 估算结果及太湖分区图: (a) 200410; (b) 200810; (c) 201004; (d) 201101; (e) 201103; (f) 太湖分区

Fig. 6 Results of $\left[a_{\mathrm{CDOM}} / a_{\mathrm{t}}\right]$ (412) estimated using MODIS images and the subarea of Lake Taihu:

(a) 200410; (b) 200810; (c) 201004; (d) 201101; (e) 201103; (f) subarea of Lake Taihu

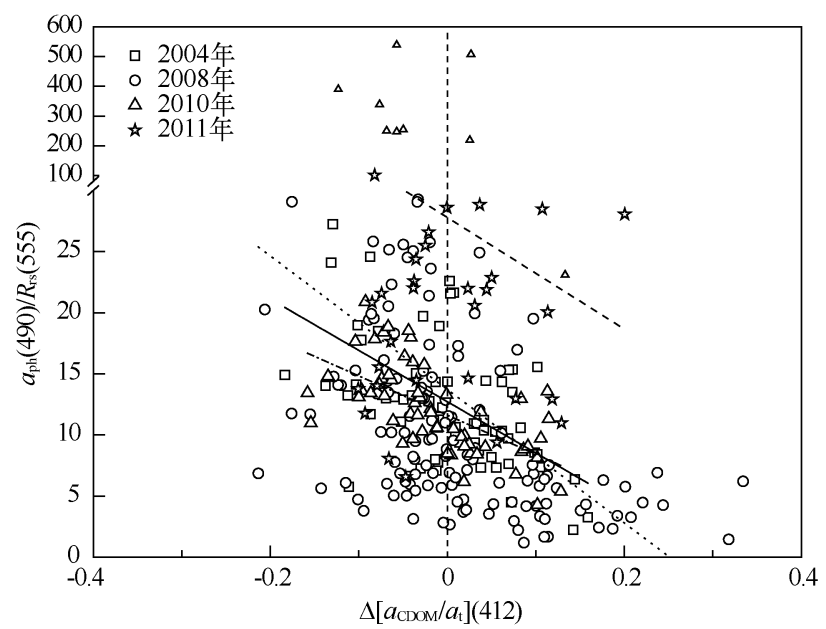

图 $7 a_{\mathrm{ph}}(490) / R_{\mathrm{rs}}(555)$ 与 $\Delta\left[a_{\mathrm{CDOM}} / a_{\mathrm{t}}\right](412)$ 的关系

Fig. 7 Relationship between $a_{\mathrm{ph}}(490) / R_{\mathrm{rs}}(555)$ and $\Delta\left[a_{\mathrm{CDOM}} / a_{\mathrm{t}}\right](412)$
比较大, 并且 $\left[a_{\mathrm{CDOM}} / a_{\mathrm{t}}\right](412)$ 的值出现高 估现象.

分析水体三组分 (纯水除外) 的吸收 比例, 以探寻不同组分在水体吸收中的主 导因子. 分析 $\operatorname{CDOM}\left(a_{\mathrm{CDOM}}(412)\right)$ 、色素 $\left(a_{\mathrm{ph}}(412)\right)$ 和非色素 $\left(a_{\mathrm{d}}(412)\right)$ 吸收系数 比例的变化发现 (图 $8 \mathrm{a}), a_{\mathrm{ph}}(412)$ 所占的 比例绝大部分集中在 $30 \%$ 之内, 所占比例 最小; $a_{\mathrm{CDOM}}(412)$ 在水色因子总吸收所占 的比例在 $0 \sim 0.75$ 之间变化; 而 $a_{\mathrm{d}}(412)$ 比例变化范围为 $0.25 \sim 1.00$. 说明非色素 物质在太湖水体中所占比例较大, 是影响 CDOM 吸收的主要因子. 建立 $a_{\text {CDOM }}(412)$ 和 $a_{\mathrm{d}}(412$ ) 所占比例之间的关系 (图 $8 \mathrm{~b}$ ) 发现, 两者呈较好的负相关关系, 表明太湖 CDOM 和悬浮泥沙占主导地位, 且两者关 系稳定 $\left(R^{2}=0.54\right)$. 

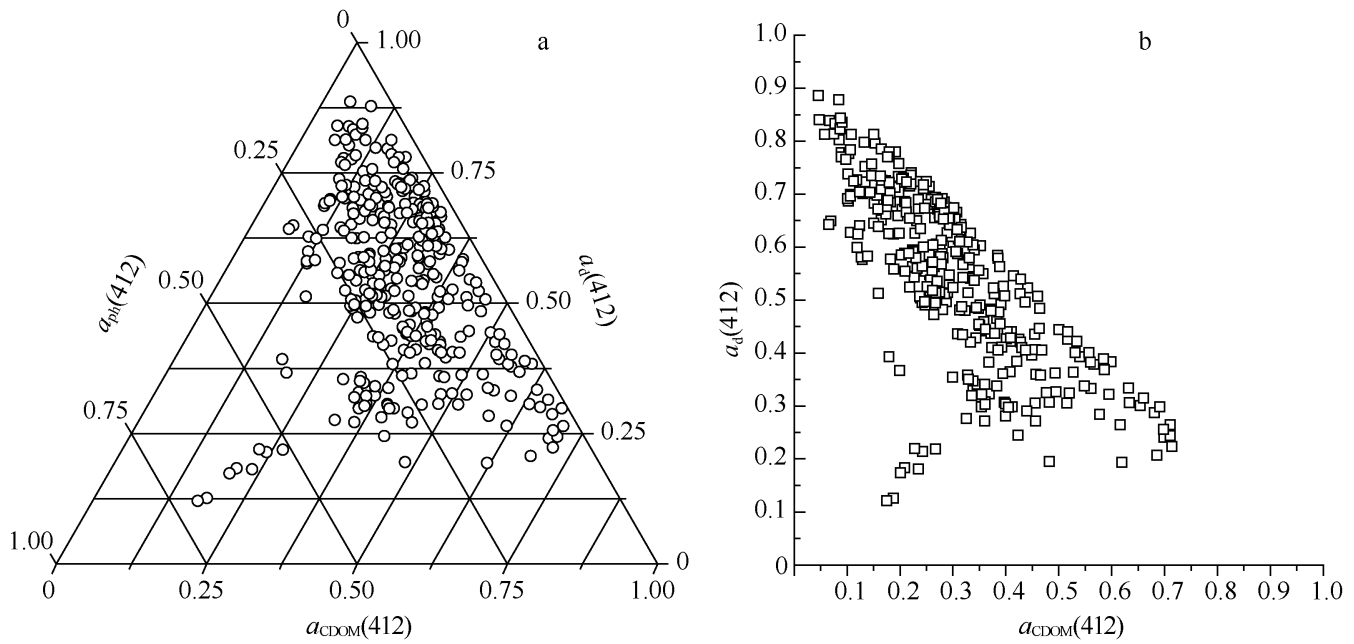

图 $8 \operatorname{CDOM}\left(a_{\mathrm{CDOM}}(412)\right)$ 、色素 $\left(a_{\mathrm{ph}}(412)\right)$ 和非色素 $\left(a_{\mathrm{d}}(412)\right)$ 吸收系数在水色因子 总吸收中占的比例 $(\mathrm{a}) ; a_{\mathrm{CDOM}}(412)$ 和 $a_{\mathrm{d}}(412)$ 所占比例之间的关系 (b)

Fig. 8 The contributions of total absorption of water variations of $a_{\mathrm{CDOM}}(412), a_{\mathrm{ph}}(412)$ and $a_{\mathrm{d}}(412)$ (a); relationship of the proportions between $a_{\mathrm{CDOM}}(412)$ and $a_{\mathrm{d}}(412)(\mathrm{b})$

\section{4 结论}

基于 200410、200810、201004、201101 和 201103 的 5 期现场数据,讨论分析太湖有色溶解有机物对水体总 吸收的贡献 $\left(\left[a_{\mathrm{CDOM}} / a_{\mathrm{t}}\right](412)\right)$ 的变化特征,并利用高光谱数据估算太湖 $\left[a_{\mathrm{CDOM}} / a_{\mathrm{t}}\right](412)$, 评价 $\left[a_{\mathrm{CDOM}} / a_{\mathrm{t}}\right]$ (412) 的遥感估算精度.

2011 年 $\left[a_{\mathrm{CDOM}} / a_{\mathrm{t}}\right]$ ( 412) 的均值最大,201004 期均值最小. 对于竺山湾和梅梁湾来说, 竺山湾水体对太 湖 $\left[a_{\mathrm{CDOM}} / a_{\mathrm{t}}\right](412)$ 的贡献较大, 而梅梁湾 $\left[a_{\mathrm{CDOM}} / a_{\mathrm{t}}\right]$ (412) 均值与整个太湖基本相等. 考虑到悬浮泥沙、浮 游植物对 CDOM 的影响, 应用多元线性模型反演 $\left[a_{\mathrm{CDOM}} / a_{\mathrm{t}}\right]$ ( 412$)$, 反演精度较高 $\left(n=333, R^{2}=0.48\right.$, RMSE $=34.60 \%)$.

浮游色素的吸收对 $\left[a_{\mathrm{CDOM}} / a_{\mathrm{t}}\right]$ (412) 遥感估算的影响造成 $\left[a_{\mathrm{CDOM}} / a_{\mathrm{t}}\right](412)$ 的值被低估, 而悬浮泥沙的 吸收对 $\left[a_{\mathrm{CDOM}} / a_{\mathrm{t}}\right](412)$ 遥感估算的影响使得 $\left[a_{\mathrm{CDOM}} / a_{\mathrm{t}}\right](412)$ 的值被高估. 太湖水体 CDOM 和非色素物质 在水色要素总吸收中占主导地位,并且悬浮泥沙是影响 CDOM 吸收的主要原因.

CDOM 与悬浮泥沙具有相似的吸收特性, 但 CDOM 没有散射特征; CDOM 在 $440 \mathrm{~nm}$ 处吸收强烈, 与浮游 植物色素吸收峰重合, 影响了浮游植物浓度的遥感反演精度 ${ }^{[13]}$, 但浮游植物色素在 $670 \mathrm{~nm}$ 处有明显的菼光 峰. 因此, 在以后的研究中, 应充分考虑悬浮泥沙的后向散射特征和浮游植物色素的苂光特性, 才能精确提 取 II 类水体的 CDOM.

致谢: 在实验过程中得到了饶家旺、赵晨露、王泽人、王长风、齐琳、苑惠丽、林珊、项文华等人的帮助, 并且在 论文的书写和修改中得到了苏文同学的帮助,在此一并感谢!

\section{5 参考文献}

[ 1 ] Morel A, Prieur L. Analysis of variation in ocean color. Limnology and Oceanography, 1977, 22 (4) : 709-722.

[ 2 ] Keith DJ, Yoder JA, Freeman SA. Spatial and temporal distribution of coloured dissolved organic matter (CDOM) in Narragansett Bay, Rhode Island: Implications for phytoplankton in coastal waters. Estuarine, Coastal and Shelf Science, 2002, 55 : $705-717$.

[ 3 ] Bélanger S, Babin M, Larouche P. An empirical ocean color algorithm for estimating the contribution of chromophoric dis- 
solved organic matter to total light absorption in optically complex waters. Journal of Geophysical Research, 2008, 113: $\mathrm{C} 04027$.

[ 4 ] Bricaud A, Morel A, Prieur L. Absorption by dissolved organic matter of the sea (yellow substance) in the UV and visible domains. Limnology and Oceanography, 1981, 26: 43-53.

[ 5 ] Sieburth J, Jensen A. Studies on algal substances in the sea. II. The formation of gelbstoff (humic material) by exudates of phaeophyta. Journal of Experimental Marine Biology and Ecology, 1969, 3 : 275-289.

[ 6] Gagosian RB, Stuermer DH. The cycling of biogenie compounds and their diagenetically, transformed products in seawater. Marine Chemistry, 1977, 5(4/5/6) : 605-632.

[ 7 ] Coble PG. Marine optical biogeochemistry: the chemistry of ocean color. Chemical Reviews, 2007, 107: $402-418$.

[ 8 ] Del Castillo CE, Miller RL. On the use of ocean color remote sensing to measure the transport of dissolved organic carbon by the Mississippi River Plume. Remote Sensing of Environment, 2008, 112(3) : 836-844.

[ 9 ] Reche I, Pace ML, Cole JJ. Modeled effects of dissolved organic carbon and solar spectra on photobleaching in lake ecosystems. Ecosystems, 2000, 3: 419-432.

[10] Prieur L, Sathyendranath S. An optical classification of coastal and oceanic waters based on the specific spectral absorption curves of phytoplankton pigments, dissolved organic matter, and other particulate materials. Limnology and Oceanography, 1981, 26: 671-689.

[11] Alison BB, James NK. The relative importance of chlorophyll and colored dissolved organic matter ( CDOM) to the prediction of the diffuse attenuation coefficient in shallow estuaries. Estuaries and Coasts, $2005,28(5)$ : 643-652.

[12] 潘德炉,马荣华. 湖泊水质遥感的几个关键问题. 湖泊科学,2008,20(2):139-144.

[13] 马荣华,段洪涛,唐军武等. 湖泊水环境遥感. 北京:科学出版社,2010:31-32.

[14] 段洪涛,马荣华,孔维娟等. 太湖沿岸水体 CDOM 吸收光谱特性. 湖泊科学, 2009,21(2):242-247.

[15] 马荣华, 戴锦芳, 张运林. 东太湖 CDOM 吸收光谱的影响因素与参数确定. 湖泊科学, 2005,17(2):120-126.

[16] 张运林, 吴生才, 秦伯强等. 太湖梅梁湾有色可溶性有机物对光的吸收. 中国环境科学, 2004,24 (4):405-409.

[17] 周虹丽, 朱建华, 李酮基等. 青海湖水色要素吸收光谱特性分析一一黄色物质、非色素颗粒物和浮游植物色素. 海 洋技术, $2005,24(2): 55-58$.

[18] 施 坤, 李云梅,王 桥等. 太湖、巢湖水体 CDOM 吸收特性和组成的异同. 环境科学, 2010,31(5):1183-1191.

[19] 杨顶田,陈伟民, 吴生才等. 湖泊中有色可溶性物质对近紫外及蓝光衰减的影响. 湖泊科学, 2003,15(3):269-274.

[20] 姜广甲, 刘殿伟, 宋开山等. 长春市石头口门水库 CDOM 的光学特性. 中国科学院研究生院学报, 2009, 26(5): $640-646$.

[21] 唐军武,田国良,汪小勇等. 水体光谱测量与分析 I: 水面以上测量法. 遥感学报,2004,8(1):37-44.

[22] Giulietta SF, James LM. Ocean optics protocols for satellite ocean color sensor validation, Revision 2. NASA/TM-2000209966, 2000.

[23] Pope RM, Fry ES. Absorption spectrum $(380-700 \mathrm{~nm})$ of pure water, II. Integrating cavity measurements. Applied Optics, 1997,36 : 8710-8723.

[24] Smith RC, Baker KS. Optical properties of the clearest natural waters $(200-800 \mathrm{~nm})$. Applied Optics, 1981,32 : $177-184$.

[25] 张运林, 黄群芳, 马荣华等. 基于反射率的太湖典型湖区溶解性有机碳的反演. 地球科学进展, 2005, 20 (7): $772-777$.

[26] 曹 静, 刘小军, 汤 亮等. 稻麦适宜氮素营养指标动态的模型设计. 应用生态学报,2010,21(4):359-364. 